\title{
Dynamic Parameters Optimization and Kinematic Analysis of Mechanical Treadmill
}

\author{
Qin $\mathrm{Li}^{1, \mathrm{a}}$ \\ ${ }^{1}$ Zhuhai College of Jilin University, Zhuhai, China \\ alubianinfo@foxmail.com
}

Keywords: Mechanical Treadmill; Kinematic Analysis; Dynamic Parameters

\begin{abstract}
With the gradual improvement of health concepts, people increasingly pay attention to their own health problems, more and more people want to be able to keep abreast of their own health. Small footprint, simple operation, easy control makes treadmill become the most common indoor fitness equipment. This paper introduces the treadmill significance and purpose of a health monitoring system, described the treadmill research status and development trends at home and abroad, and some brands of treadmill structure and patents were introduced and then on human health research status and development trend monitoring were analyzed, a clear research objectives and research. In this paper, the kinematics treadmill and reliability is analyzed. By kinematic analysis, obtained treadmill gradient changes the movement of the curve to improve the accuracy grade control.
\end{abstract}

\section{Introduction}

With the development of the times, people's material life in the continuous improvement of living standards have gradually been met, then people began to pursue a healthy lifestyle, pay more attention to the care of the body, more physical exercise. One important tool for fitness equipment as physical exercise, more and more people's favorite. Running is a human instinct, is a popular international sport by the medical profession and spoke highly of aerobic fitness activities, is known to keep a person physically and mentally the most effective and the most scientific way to fitness in strengthening limb muscles and bones function, improve heart and lung function, weight loss has a significant effect. With the development of society, on the one hand people need a good physique to adapt to the needs of work, on the other hand due to some objective factors, climatic constraints, the environment, space and other opportunities for us to go for a run of fewer outdoor, indoor jogging should need era fashion, the application has become the preferred method of exercise treadmill people. From a scientific point of view, on the treadmill walking or running on the action appearance almost walk or run on the same ground, but the real hard look at the human body, it is more than walking or running on land eliminates the kick action, so that movement feel more comfortable. So treadmill into the home or the gym is a matter of course [1].

Since the 1980s, with the development of economy and technology, people's living standards improve, people began to get more physical exercise, treadmill also began to pop up. Domestic and foreign companies starting to focus research on the treadmill, after three years of continuous design and innovation, the development of the treadmill replacement. Mechanical treadmill treadmill is an earlier generation, the transmission system is composed of a conveyor belt cover, which is fixed at both ends of two shafts to tighten the belt, there is a roller shaft and connected to a switch and an adjustable mounting bracket structure, rely mainly on foot runners and running friction belt drive to run. This treadmill is simple, durable, without ity.

\section{The Good Effects of Treadmill for Human Health}

Running is a long history, wide mass, the larger the value of an exercise fitness "as early as two thousand years ago in ancient Greece, this rock is carved the words, If you want strong, running it! If you think fit, running it, If you want smart, run. Today, millions of runners has become a well-attended masses fitness movement, welcomed by the people, had been regarded as the best of 
health and fitness," the most perfect exercise. "

Running can protect the heart, the heart is an important organ of the human body easy to aging, the normal function of the heart has a direct effect on other internal organs function. Keep running exercise, can prevent the occurrence of coronary heart disease, coronary heart disease for those who already have a therapeutic effect. Can improve the function of the heart's ability to adapt the structure, due to frequent intense myocardial contraction and relaxation, coronary dilation, blood flow increases coronary circulation, thereby improving the oxygen supply to the myocardium state, improve the metabolism of the myocardium. Easy to get middle-aged ischemic heart disease, coronary artery blockage caused due to myocardial ischemia, caused by angina to myocardial infarction. Coronary jogging exercise can maintain good blood circulation, long-running exercise were not due to age and coronary artery narrowing, ensure adequate myocardial blood supply, which can prevent all kinds of heart disease, maintain good cardiac function. Running is a whole body movement, the whole body can accelerate blood circulation, adjust the distribution of systemic blood, eliminate congestion phenomenon. Through lower limb movement, pushing the body to move forward at the same time, driven by strong venous return to the heart, reduce and pelvic venous congestion, venous thrombosis prophylaxis. While the cardiac muscle fibers thicken, contractility, the volume increases, thereby enhancing the ability of the heart [2].

Running helps improve lipid metabolism, reduce hyperlipidemia, reduce high serum cholesterol levels, control of body weight, an excellent way to lose weight, control the development of atherosclerosis, but also microvascular organization increase in the number open. Due to strengthen heart function, so that more oxygen to the body tissues, thus improving tissue metabolism. Since the function of the central nervous system regulation to improve, so that arterial blood pressure decreases. Running training can also make the blood system in leukocytes. Erythrocytes and increased hemoglobin and enhance endocrine function, improve the body's resistance. Running can adjust the cerebral cortex excitatory and inhibitory, useful to improve the function of the nervous system, mental fatigue, prevent neurasthenia. You can also regulate the body's internal balance, regulating mood, pick yourself up, people often practice long-distance running is a deeper experience, there are significant health cardiac effects.

\section{The Kinematic Analysis and Improvement Analysis of the Treadmill}

In treadmill slope adjustment process, because of its slope with the amount of projection of the push rod linear change is unknown, but also at an arbitrary position to be changed by the same slope change stretch amount is unknown, the kinematics analysis of the treadmill obtained at the various locations of displacement, can provide the basis for grade control.

Treadmill slope adjustment diagram shown in Fig. 1. Which, AB bar linkage for collapse, CD rod for treadmill chassis (ie, jogging sets), EF lever for lifting the motor putter. When changing the amount of lift motor plunger extends, the base chassis ground of the treadmill and the angle will change, i.e. $\alpha$ will change as the push rod protruding amount varies.

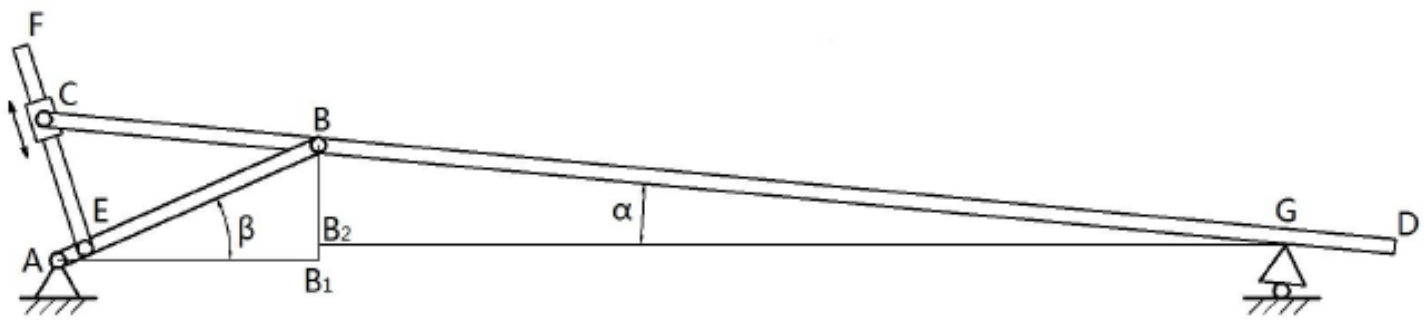

Fig. 1 Slope adjustment mechanism

Set $A B=a, B C=b, B G=c, B E=e$, the angle between the club and the ground for $C D$, the angle $\beta$ between the club and the ground as $\mathrm{AB}$. A point and the horizontal height is $d_{1}$ the height of the G-spot and the horizontal $d_{2}$. C since the sleeve slides on EF, the position is changed, assuming $\mathrm{CE}=\mathrm{x}$. Figure 3.1 by the geometric relationship can get the following set of equations: 


$$
\left\{\begin{array}{c}
\angle A B C=\alpha+\beta \\
\cos (\alpha+\beta)=\frac{b^{2}+e^{2}-x^{2}}{2 b e} \\
\alpha \sin \beta+d_{1}=c \sin \alpha+d_{2}
\end{array}\right.
$$

You can get an expression about the $\beta$ and $\alpha$ :

$\mathrm{X}$ can be obtained about $\alpha$ on the expression:

$$
\beta=\arcsin \left(\frac{c \sin \alpha+d_{2}-d_{1}}{2 b e}\right)
$$

$$
x=\sqrt{\left.b^{2}+e^{2}-2 b e \sqrt{a^{2}-\left(c \sin \alpha+d_{2}-d_{1}\right)^{2}} \cdot \sqrt{1-\sin ^{2} \alpha}-\sin \alpha\left(c \sin \alpha+d_{2}-d_{1}\right)\right) / a}
$$

Therefore, to get the desired slope $\alpha$, as long as the value of the distance $\mathrm{G}$ and the lever position $\mathrm{AB}, \mathrm{BC}$ lever, $\mathrm{BG}$ rod, $\mathrm{E}$ the point on $\mathrm{AB}, \mathrm{A}$ point with the horizontal plane and the horizontal distance to take a given, according to the above you can find the value of $\mathrm{x}$ in the expression.

By the above formula $\cos (\alpha+\beta)$ may be known values between 0-1, CE major distance $\mathrm{x}$ from the rod position determining the length of $\mathrm{AB}$ and $\mathrm{BC}$ at point $\mathrm{E}$ where the rod. Since the $\mathrm{BC}$ portion of the rod indicates jogging station, by the length of the actual situation found that this part is relatively fixed, little change, so the distance $x \mathrm{CE}$ mainly by point $\mathrm{E}$ in $\mathrm{AB}$ lever position to decide [3].

This paper selected $A B=300 \mathrm{~mm}, \mathrm{BC}=320 \mathrm{~mm}, \mathrm{BG}=1350 \mathrm{~mm}, \mathrm{~d} 1=65 \mathrm{~mm}, \mathrm{~d} 2=80 \mathrm{~mm}$. BE were taken $220 \mathrm{~mm}, 240 \mathrm{~mm}, 260 \mathrm{~mm}$, while $280 \mathrm{~mm}$, four sets of curves can be obtained CE slope and distance $\mathrm{x}$.
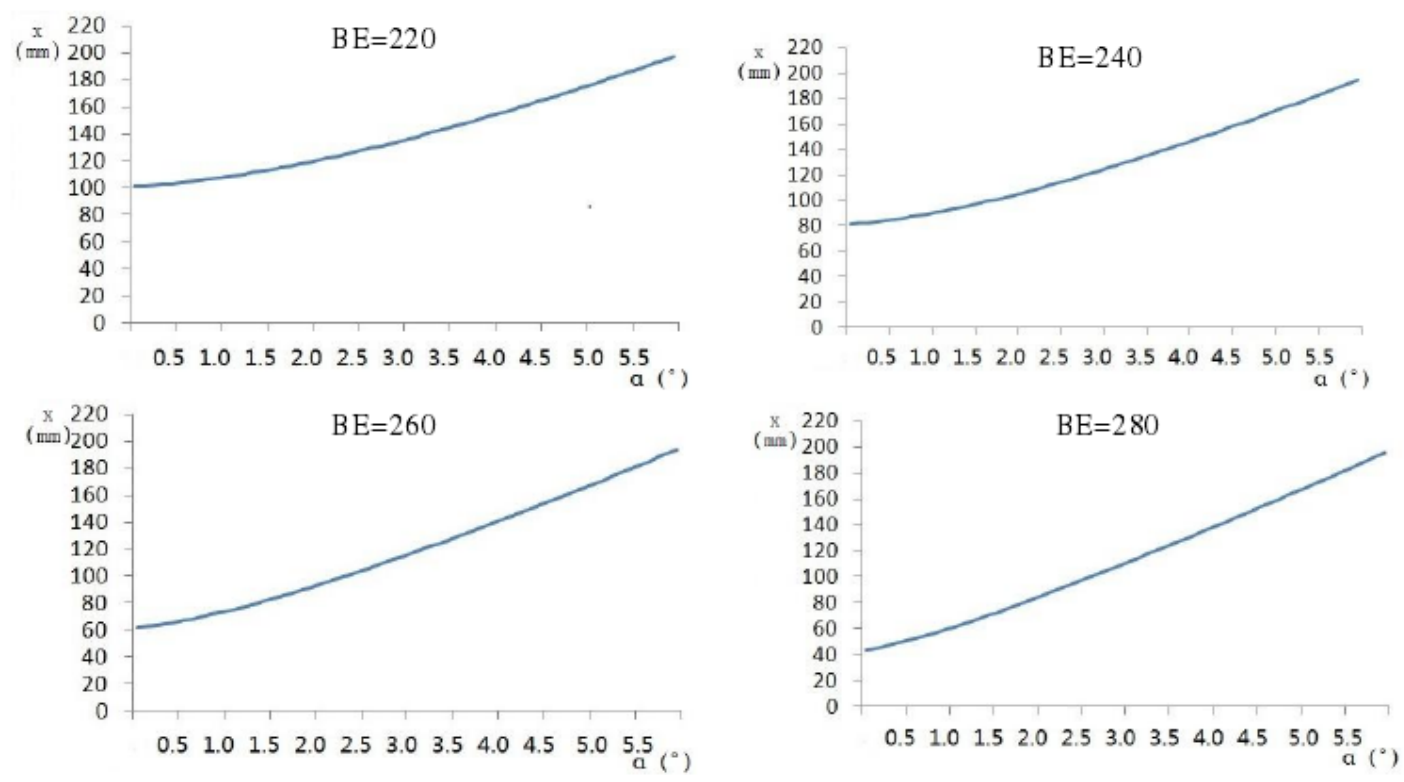

Fig. 2 Curve slope and different values of $\mathrm{x}$ when the rod BE

FIG four curves can be found in, when close to $0^{\circ}$, the initial distance CE is determined by the point $E$ in the $A B$ rod position, when the slope close to $6^{\circ}$ when, $C E$ distance are about 200mm; values $\mathrm{BE}$ rod the larger, it is equivalent to $\mathrm{E}$ point closer to point $\mathrm{A}$, the slope and the distance $\mathrm{X}$ CE linear relationship [4].

Slider pendulum mechanism as the treadmill slope adjustment mechanism to achieve change in the slope of the function of the movement, while the organization is relatively simple, and easy to control.

Then, using software simulation approach to transport treadmill kinematic analysis. In the Pro / e assembly mode, the required parts in order according to the constraint between them assembled, and in the front of the chassis to take a little PNT1 [5], at the bottom of the caster take a little PNT0. 


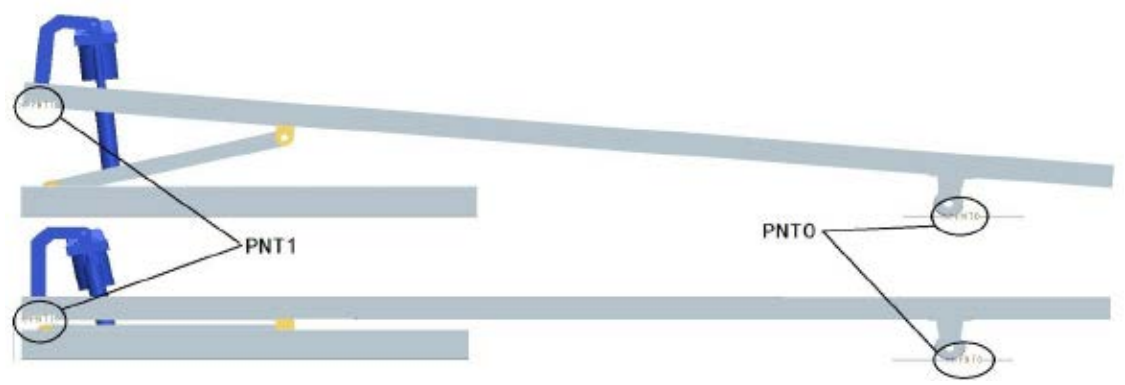

Fig. 3 Elevating mechanism assembly drawing

After assembly is completed, the application will switch to the agency (E) mode, and lift motor is defined, the definition of the speed of the plunger to push away as $1 \mathrm{~mm} / \mathrm{s}$, the numerical value and the time to push the process of equality, you can use. And the horizontal and vertical distances PNT0 and PNT1 indirect measurements, shown in Fig. 3. Export the measured data, after processing, you can get the slope of the curve with the process of putting the push.

\section{Conclusion}

Treadmill is a fitness equipment for public health and it has a small footprint, safe and easy, more and more are wanted for simple fitness activities in the home fitness accepted and positive for fitness equipment.

Although this study mechanical body and human health monitoring treadmill was analyzed. As a result of the athletes during exercise, treadmill running boards bear the brunt of the load, so it will support the running board supports the alternating stress, fatigue damage and the running speed of the belt may be affected. In future, it hope to be analyzed in this context and verify its reliability.

\section{Acknowledgements}

Fund project: Zhu hai college of JiLin University hundred projects(NO.4149010122).

\section{References}

[1] MarkVella.: Jnatomy for Strength and Fitness Training, Vo1. 4 (2010) No. 26, p.20-29.

[2] Mclntire KL, Asher MA, Burton DC,Liu W, Wang Fucheng: J Spinal DISord Tech, Vo1. 4 (2010) No. 5, p. 411-419.

[3] Fok CL, Li Changhong, etc.: First International Conference on Advances in Future Internet, Vo1. 34 (2009) No. 11, p. 1608-1618.

[4] Sun Qibo: Journal of Anhui University of Technology, Vo1. 23 (2010) No. 26, p. 786-793.

[5] Liu Jie, Li Shan: Diagnostic and statistical Manual of mental disorders, Vo1. 31 (2007) No.27, p. 260-264. 